\title{
Ulinastatin Alleviates Neurological Deficiencies Evoked by Transient Cerebral Ischemia via Improving Autophagy, Nrf-2-ARE and Apoptosis Signals in Hippocampus
}

\author{
Xiao-Ming JIANG ${ }^{1}$, Jing-Hai HU ${ }^{2}$, Lu-Lu WANG ${ }^{3}$, Chi MA ${ }^{4}$, Xu WANG ${ }^{5}$, Xiao-Liang LIU ${ }^{1}$ \\ ${ }^{1}$ Department of Emergency Medicine, The First Hospital of Jilin University, Changchun, Jilin, \\ China, ${ }^{2}$ Department of Urology, The First Hospital of Jilin University, Changchun, Jilin, China, \\ ${ }^{3}$ Cardiovascular Center for Diagnostics and Treatment, The First Hospital of Jilin University, \\ Changchun, Jilin, China, ${ }^{4}$ Department of Brain Tumor Surgery, The First Hospital of Jilin \\ University, Changchun, Jilin, China, ${ }^{5}$ Department of Neurology and Neuroscience Center, The First \\ Hospital of Jilin University, Changchun, Jilin, China
}

Received October 17, 2017

Accepted February 2, 2018

On-line May 10, 2018

\section{Summary}

Ulinastatin [or called as urinary trypsin inhibitor (UTI)] plays a role in regulating neurological deficits evoked by transient cerebral ischemia. However, the underlying mechanisms still need to be determined. The present study was to examine the effects of UTI on autophagy, Nrf2-ARE and apoptosis signal pathway in the hippocampus in the process of neurological functions after cerebral ischemia using a rat model of cardiac arrest (CA). CA was induced by asphyxia followed by cardiopulmonary resuscitation (CPR) in rats. Western blot analysis was employed to determine the expression of representative autophagy (namely, Atg5, LC3, Beclin 1), p62 protein (a maker of autophagic flux), and Nrf2-ARE pathways. Neuronal apoptosis was assessed by determining expression levels of Caspase- 3 and Caspase- 9 , and by examining terminal deoxynucleotide transferase-mediated dUTP nick-end labeling (TUNEL). The modified neurological severity score (mNSS) and spatial working memory performance were used to assess neurological deficiencies in CA rats. Our results show that CA amplified autophagy and apoptotic Caspase-3/Caspase-9, and downregulated Nrf2-ARE pathway in the hippocampus CA1 region. Systemic administration of UTI attenuated autophagy and apoptosis, and largely restored Nrf2-ARE signal pathway following cerebral ischemia and thereby alleviated neurological deficits with increasing survival of CA rats. Our data suggest that UTI improves the worsened protein expression of autophagy and apoptosis, and restores Nrf2-ARE signals in the hippocampus and this is linked to inhibition of neurological deficiencies in transient cerebral ischemia. UTI plays a beneficial role in modulating neurological deficits induced by transient cerebral ischemia via central autophagy, apoptosis and Nrf2-ARE mechanisms.

\section{Key words}

Ulinastatin • Cardiac arrest • Cerebral ischemia • Autophagy • Apoptosis $\bullet$ Cardiopulmonary resuscitation

\section{Corresponding authors}

X. Wang and X.-L. Liu, Department of Neurology and Neuroscience Center, Department of Emergency Medicine, The First Hospital of Jilin University, 71 Xinmin Street, Changchun, Jilin 130021, China. E-mail: wangxxu2016@163.com

\section{Introduction}

During cardiac arrest (CA) occurs, blood flow and oxygen delivery are abruptly halted and this leads to systemic ischemic injury in various organs including brain (Nolan et al. 2008). Although cardiopulmonary resuscitation (CPR) is applied inadequate blood flow and tissue oxygen delivery still persist due to myocardial dysfunction, hemodynamic instability and microvascular dysfunction. Thus, it is important to study signal pathways involved in neuroprotective effects and further determine biological agents that can attenuate damages 
evoked by transient cerebral ischemia.

Ulinastatin, also called urinary trypsin inhibitor (UTI), is a glycoprotein that acts as a trypsin inhibitor (Inoue and Takano 2010). It can be derived from urine or synthetically produced and conventionally effective in treatment of acute and chronic pancreatitis, toxic shock and sepsis etc. (Inoue and Takano 2010, Wang et al. 2013, Karnad et al. 2014). It has also been reported that UTI suppresses neutrophil accumulation and activity. The genes and proteins regulated by UTI are implicated in the inflammatory process (Inoue et al. 2008). Therefore, UTI is not just a protease inhibitor, but can also prevent inflammation and cytokine-dependent signaling pathways. In preclinical and clinical studies, UTI has been reported to protect against acute lung injury, graft ischemia/ reperfusion injury, renal failure after cardiopulmonary bypass, severe burn injury and septic shock (Inoue and Takano 2010). Our recent study further demonstrated that systemic administration of UTI can attenuate amplification of central pro-inflammatory cytokine (PIC) signal pathway in a rat model of cerebral ischemia induced by CA thereby leading to improvement of neurological severity score, brain tissue edema and survival (Jiang et al. 2016). Consistent with this result, UTI has been reported to attenuate neuronal nuclear factor (NF)- $\mathrm{kB}$ and PICs and further improve neural functions, and inhibition of PICs leads to downregulation of apoptotic signals (Sui et al. 2014, Liu et al. 2016, Xing and $\mathrm{Lu}$ 2016). These data indicate the beneficial role played by UTI in alleviating cerebral ischemia reperfusion injury. Nonetheless, the underlying mechanisms leading to the effects of UTI on ischemic insults still need to be determined.

As an alternative cell death program, autophagy is considered as an important non-apoptotic cell death pathway (Blumberg et al. 1997, Edinger and Thompson 2004). Autophagy can be stimulated in both adult and neonatal animals, and it also contributes to ischemic neuronal injury (Northington et al. 2011). Prior studies have frequently shown that the inhibition of autophagy signal protects cell death caused by ischemic brain injury (Koike et al. 2008, Lu et al. 2015). Nonetheless, there is lacking of evidence showing that UTI influences autophagy signal in contribution to improvement of cerebral ischemia following CA. Evidence provided by such line of experiments would be significant for a clinical application of UTI.

In light of effective autophagy signal responsible for UTI in response to cerebral ischemic conditions, in the present study, we determined the protein expression levels of representative autophagy such as Atg5, LC3 (including cytosolic LC3 and autophagosomes bound part of LC3) and Beclin 1 in the hippocampus CA1 of rats after CA induced-transient cerebral ischemia. In addition, we determined the levels of p62 protein [also called sequestosome 1 (SQSTM1), a maker of autophagic flux]. We postulated that CA can upregulate Atg5, LC3 and Beclin 1 and this leads to a decrease in the levels of p62 protein. We hypothesized that UTI attenuates the protein expression of Atg5, LC3 and Beclin 1 in hippocampus CA1 region of ischemic rats and thereby recovers the levels of $\mathrm{p} 62$ protein.

Nuclear factor (erythroid-derived 2)-like 2 (Nrf2, also known as NFE2L2) is a transcription factor and as a basic leucine zipper protein it regulates the expression of antioxidant proteins that protect against oxidative damage triggered by injury and inflammation (Moi et al. 1994). Numerous drugs that stimulate the Nrf2 pathway have been reported for treatment of diseases caused by oxidative stress (Gold et al. 2012). Thus, in this study we measured the levels of 8-isoprostaglandin F2 $\alpha$ (8-iso PGF2 $\alpha$, a product of oxidative stress) in hippocampus in order to determine the engagement of oxidative stress, and Nrf2-antioxidant response element (ARE) pathway in the hippocampus following CA. We hypothesized that UTI upregulates Nrf2 signal and Nrf2-regulated NADPH quinone oxidoreductase-1 (NQO1), and this decreases oxidative production and attenuates apoptotic signal in hippocampus CA1 of ischemic animals. Our overall hypothesis was that UTI plays a beneficial role in modulating neurological deficits evoked by cerebral ischemia via improving central autophagy, Nrf2-ARE and apoptosis mechanisms.

In general, in the pathophysiological process of diseases apoptosis and autophagy play an important role, i.e. the prior study has suggested that these signals are engaged in transient cerebral ischemia (Cui et al. 2016). UTI plays a beneficial role in regulating neurological dysfunctions evoked by cerebral ischemia, and PIC signal and apoptosis mechanisms are likely engaged in the effects (Sui et al. 2014, Jiang et al. 2016, Liu et al. 2016, Xing and Lu 2016). However, the effects of UTI on autophagy and Nrf2 signals are lacking. Thus, in the present study we have emphasized engagement of autophagy and Nrf2. In order to better assess the effects of UTI, we have also examined its effects on apoptosis. 


\section{Materials and Methods}

All the animal procedures were approved by the Institutional Animal Care \& Use Committee of Jilin University, which were in compliance with the Guideline for the Care and Use of laboratory Animals of the International Association for the Study of Pain. Male Sprague-Dawley rats (200-300 g; 6-8 weeks) were used in our experiments.

The ischemia was produced in the CA model induced by asphyxia. Briefly, rats were anesthetized with an isoflurane-oxygen mixture (2-5\% isoflurane in $100 \%$ oxygen). An endotracheal tube was first inserted and attached to a ventilator. The ventral tail artery was cannulated to monitor systemic arterial pressure. The right jugular vein was cannulated for a continuous infusion of saline (at a rate of $0.1 \mathrm{ml} / \mathrm{h}$ ) to maintain baseline blood pressure and fluid balance using a syringe pump. Body temperature was continuously monitored and maintained at $37^{\circ} \mathrm{C}$ with a heating pad and external heating lamps. Heart activity was monitored via recording electrocardiography using electrodes placed on the skin. Asphyxia was induced by stopping mechanical ventilation and clamping the tracheal tubes at the end of expiration. Resuscitation efforts began $6 \mathrm{~min}$ following CA was induced. For this purpose, rats were orotracheally intubated for mechanical ventilation accompanied by chest compression delivered by a mechanical compressor at a rate of 200/min for $5 \mathrm{~min}$. Once spontaneous heartbeat returned, epinephrine $(2 \mu \mathrm{g})$ was administered to achieve a mean arterial blood pressure of $>80 \mathrm{~mm} \mathrm{Hg}$. Ventilation was adjusted for animals to regain spontaneous respiration and achieve normoxia. In the sham operated animals, the same surgical procedures were performed without cardiac arrest and resuscitation.

The rats were divided randomly. Group 1 $(n=12)$ : the rats received the same surgical procedures and endotracheal intubation was performed with no asphyxia and CPR. Group $2(\mathrm{n}=18)$ : CA and CPR were performed and $0.5 \mathrm{ml}$ of saline (i.p., every $12 \mathrm{~h}$ for 7 days) was given after CA. Group $3(n=25)$ : CA and CPR were carried out and 20,000 units $/ \mathrm{kg}$ body weight of UTI (i.p., every $12 \mathrm{~h}$ for 7 days) was injected after CA (Sui et al. 2014, Li X. et al. 2017). In additional group, in order to determine effectiveness of UTI 3-methyladenine (3-MA, inhibitor of autophagy; $15 \mathrm{mg} / \mathrm{kg} / \mathrm{day}$, i.p.) (Li L. et al. 2017) was injected in CA rats $(n=5)$. At the end of each experiment, the rats were sacrificed and then the hippocampus was taken out for biochemical measurements.

Seven days after the rats received all the treatments, the modified method of neurological severity score (mNSS) was used to examine neurological functions in this study as described previously (Jiang et al. 2016). Note that mNSS was generally used to assess a combination of motor, sensory, and balance functions. Neurological function was graded on a scale of $0-18$ (normal score, 0; maximal deficit score, 18). If we found that mNSS score of rats was $>0$ before CA, this indicated that the rats were abnormal and they were excluded from the experiment. It is noted that the experiments were performed in a blind manner.

Spatial working memory performance was assessed by recording spontaneous alternation performance in a Y-maze. The maze was made of grey-painted vinylchloride. Each arm was $50 \mathrm{~cm}$ long, $30 \mathrm{~cm}$ high and $10 \mathrm{~cm}$ wide and converged at an equal angle. Each rat was placed at the center of the maze and allowed to move freely through it during an 8-min period. The numbers of arm entries were recorded. An alternation was defined as entries into all arms. The percentage of alternation was calculated as

$$
\frac{\text { actual alternations }}{\text { total entered }-2} \times 100
$$

The tissues from individual rats were sampled for the analysis. In brief, the hippocampus CA1 region of the rats was removed under an anatomical microscopy. Total protein was extracted by homogenizing the hippocampus sample in ice-cold immunoprecipitation assay buffer with protease inhibitor cocktail kit. The lysates were centrifuged and the supernatants were collected for measurements of protein concentrations using a bicinchoninic acid assay reagent kit.

The levels of 8 -iso PGF2 $\alpha$ were examined using an ELISA assay kit (Promega Co., Madison, WI, USA) according to the provided description and modification. Briefly, polystyrene 96-well microtitel immunoplates were coated with affinity-purified rabbit anti-8-iso PGF2 $\alpha$ antibodies. Parallel wells were coated with purified rabbit IgG for evaluation of nonspecific signal. After overnight incubation, plates were washed. Then, the diluted samples and 8 -iso PGF2 $\alpha$ standard solutions were distributed in each plate. The plates were washed and incubated with anti-8-iso PGF2 $\alpha$ galactosidase. Then, the plates were washed and incubated with substrate solution. After incubation, the optical density was measured using an ELISA reader.

After being denatured by heating at $95^{\circ} \mathrm{C}$ in an SDS sample buffer, the supernatant samples were 
loaded onto Mini-Protean TGX Precast gels and electrically transferred to a polyvinylidene fluoride membrane. The membrane was then incubated overnight with primary antibodies: rabbit anti-Atg5, anti-LC3/B, anti-Beclin 1, anti-p62 protein, anti-Nrf2, anti-NQO1, anti-Caspase-3/Caspase-9 (cleaved form). After being fully washed, the membrane was incubated with horseradish peroxidase-linked anti-rabbit secondary antibody and visualized for immunoreactivity. All antibodies were obtained from the Abcam Co. (Cambridge, USA) and Santa Cruz Biotech (Dallas, USA). The membrane was stripped and incubated with mouse anti- $\beta$-actin to show equal loading of the protein. The bands recognized by the primary antibody were visualized by exposure of the membrane onto an X-ray film. Then, the film was scanned and the optical densities of Atg5, LC3, Beclin 1, p62 protein, Nrf2, NQO1, Caspase-3/Caspase- 9 and $\beta$-actin bands were determined using the Scion Software. Values for densities of immunoreactive bands $/ \beta$-actin band from the same lane were determined. Each of the values was then normalized to a control sample.

Neuronal apoptosis in the hippocampus CA1 region was assessed by examining terminal deoxynucleotide transferase-mediated dUTP nick-end labeling (TUNEL) staining using an in situ cell death detection kit (Roche China, Shanghai, China) according to the manufacturer's recommendations. TUNEL-positive neurons in the hippocampus were observed using a Nikon fluorescence microscope. The number of viable TUNELpositive neurons in the hippocampus was counted in the predefined area $\left(0.25 \mathrm{~mm}^{2}\right)$ at a magnification of 400 -fold (Gao et al. 2012) using Nis-Elements software.

Experimental data were analyzed using one-way repeated measures analysis of variance (ANOVA). As appropriate, Tukey's post hoc tests were used. All values were presented as mean \pm standard deviation. For all analyses, differences were considered significant at $P<0.05$. All statistical analyses were performed using SPSS for Windows version 20.0.

\section{Results}

The survival rate of 7 days was $100 \%$ (12/12 rats) in sham control rats; $60 \%$ (18/30 rats) in CA rats; $83 \%$ (25/30 rats) in CA rats injected with UTI. It is noted that the survival rate was increased when CA rats received UTI treatment as compared to CA rat without treatment. Data obtained from those survival rats were included for the analysis in this report.

Figure 1A illustrates the protein expression of representative autophagy including Atg5, LC3 band I and II (indicating cytosolic LC3 and autophagosomes bound part of LC3, respectively) and Beclin 1, and p62 protein, a maker of autophagic flux. Atg5, LC3 and Beclin 1 were upregulated and $\mathrm{p} 62$ protein was decreased in the hippocampus $\mathrm{CA} 1$ region of $\mathrm{CA}$ rats as compared with control animals $(P<0.05$, control vs. CA group; $\mathrm{n}=6$ in each group). This figure also shows that UTI significantly attenuated the amplified autophagy and recovered the levels of p62 protein $(P<0.05$, CA rats vs. CA rats with UTI; $n=6$ in CA group and $n=8$ in CA rats with UTI). Figure $1 \mathrm{~A}$ further shows that the protein expression levels of Nrf2 and Nrf2-regulated NQO1 were decreased in CA rats and UTI can restore impaired Nrf2 signal expression $(P<0.05, \mathrm{CA}$ rats vs. controls and CA rats with UTI; $n=6-8$ ). Note that expression of autophagy, Nrf2 and NQO1 was not significantly changed by UTI in control group.

In order to determine effectiveness of UTI, we also examined the protein expression levels of LC3 in CA1 region of $\mathrm{CA}$ rats $(\mathrm{n}=5)$ that were injected with 3-MA inhibiting autophagy signal. Figure 1B demonstrates that 3-MA significantly attenuated LC3, but failed to significantly alter Nrf2 and NQO1. This suggests that the effects of UTI on Nrf2 signal were likely independent of autophagy.

Figure 2A shows that 8 -iso PGF2 $\alpha$ was increased in the hippocampus of $\mathrm{CA}$ rats $(P<0.05$ vs. control rats, $\mathrm{n}=12$ in control and $\mathrm{n}=18$ in CA rats). It is noted that administration of UTI significantly attenuated increases of 8 -iso PGF $2 \alpha(P<0.05$ vs. CA rats without treatment; $\mathrm{n}=25$ in CA rats plus UTI). There were no significant differences observed in the levels of 8-iso PGF2 $\alpha$ between control group and CA group with UTI $(P>0.05)$.

In addition, Figure 2B illustrates that induction of CA significantly increased the protein expression of cleaved form of Caspase- 3 and Caspase- 9 in the hippocampus $\mathrm{CA1}$ region $(P<0.05$, control rats vs. CA rats; $\mathrm{n}=6-8$ in each group). As UTI was injected, expression of both Caspase- 3 and Caspase- 9 was significantly decreased. Note that there were no significant differences in the levels of Caspase- 3 and Caspase- 9 in control rats and CA rats that received UTI. Furthermore, Figure $2 \mathrm{C}$ shows that the number of TUNEL-positive neuronal cells was greater in the hippocampus CA1 region of CA rats than that in control rats and UTI significantly attenuated an increase of TUNEL-positive neuronal cells in CA rats. 


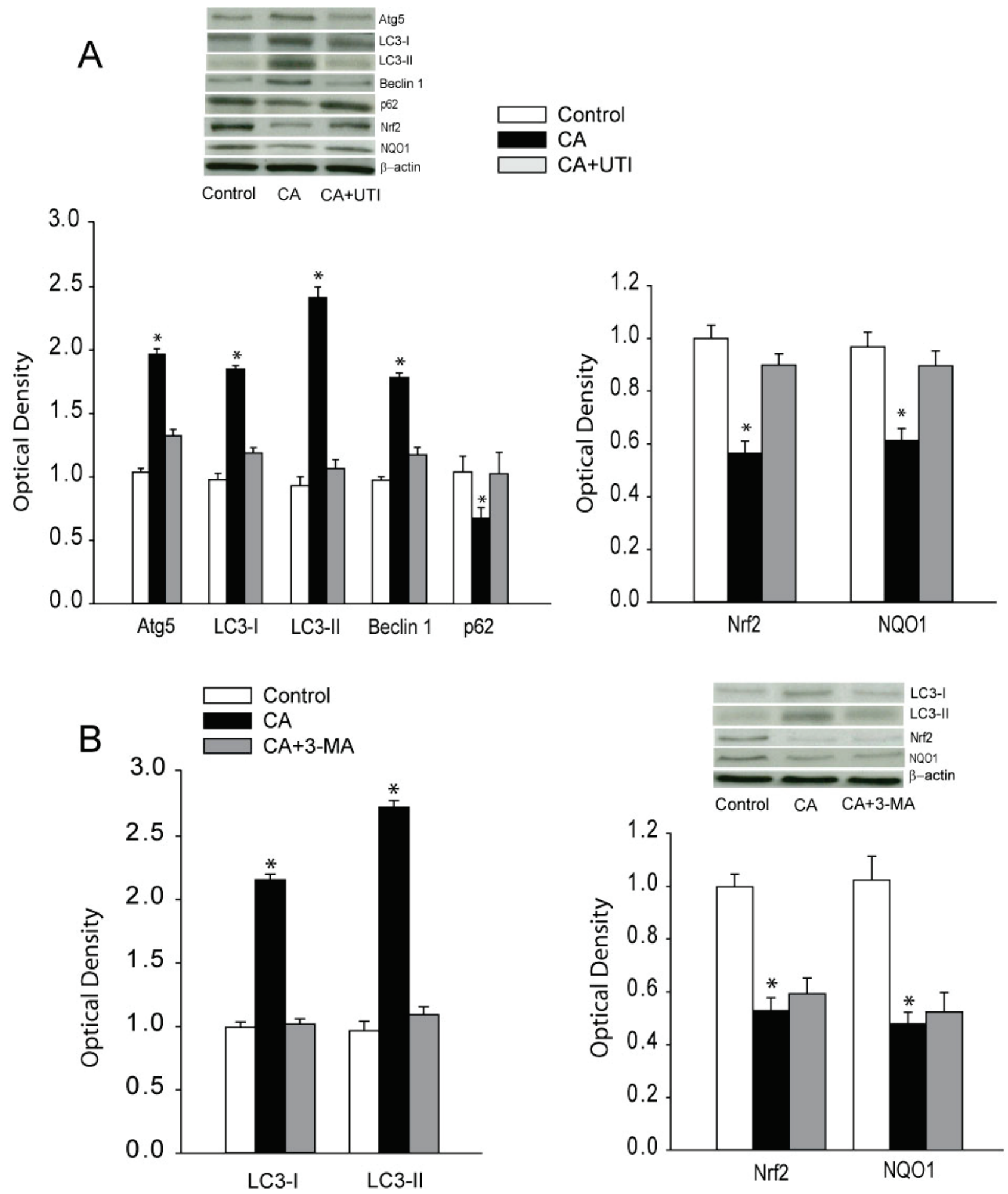

Fig. 1. Protein expression levels of autophagy and Nrf2-ARE signal. (A) Top panel and bottom panel are representative bands and averaged data: Atg5, LC3 (band I and II) and Beclin 1 were increased, and p62 protein was decreased in the hippocampus CA1 region of CA rats and UTI attenuated upregulation of autophagy and downregulation of p62 protein induced by CA. Nrf2 and NQO1 were decreased in the hippocampus of CA rats and UTI can restore impaired Nrf2 and NQO1. $* P<0.05$, CA rats vs. control rats and CA rats injected with UTI. $n=6$ in each of control and CA groups; $n=8$ in CA rats with UTI. (B) Averaged data and representative bands: 3-MA effectively attenuated LC3 (band I and II) in the hippocampus of CA rats with no significant effects on Nrf2 and NQO1. $* P<0.05$, CA group vs. control and CA plus 3-MA for LC3; and vs. control for Nrf2 and NQO1. $n=5$ in CA rats injected with 3-MA. 
A
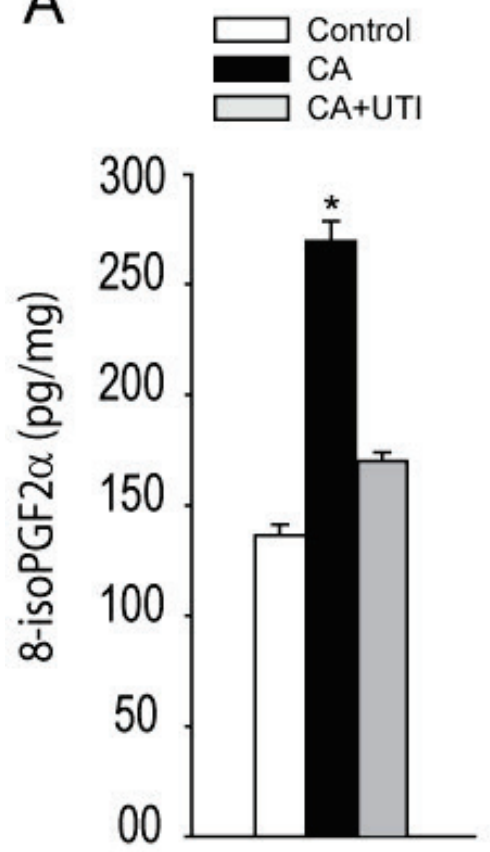

C
B
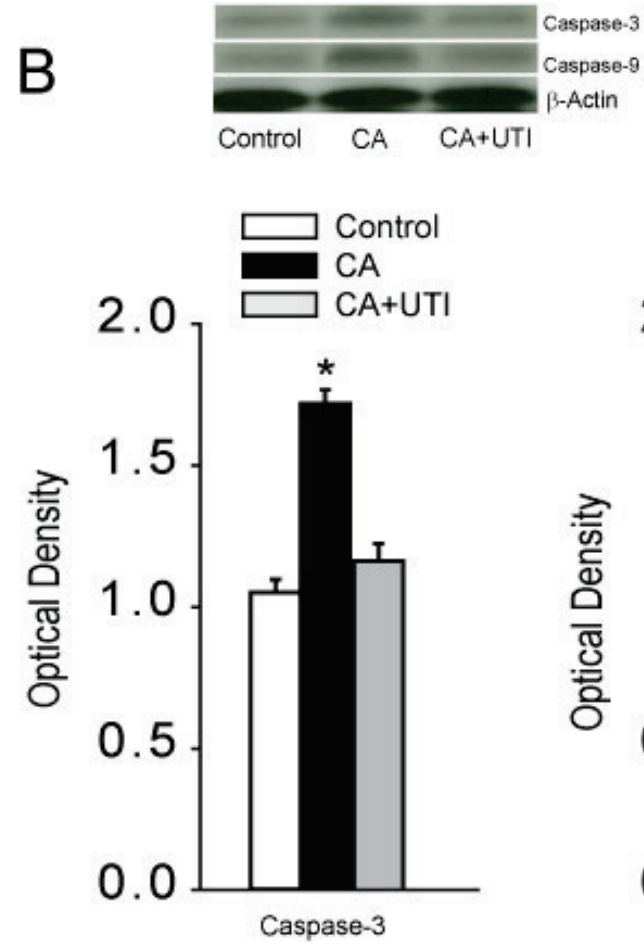

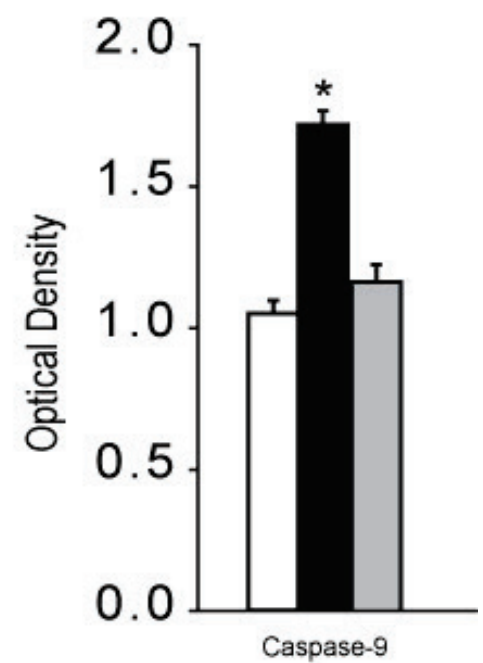

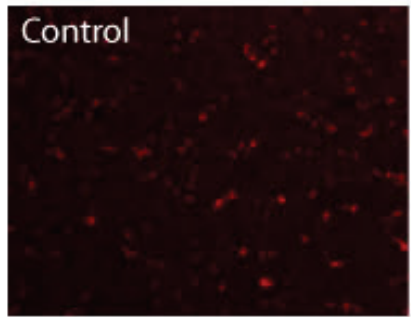

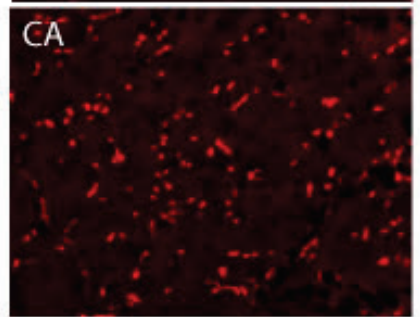

\section{CA+UTI}

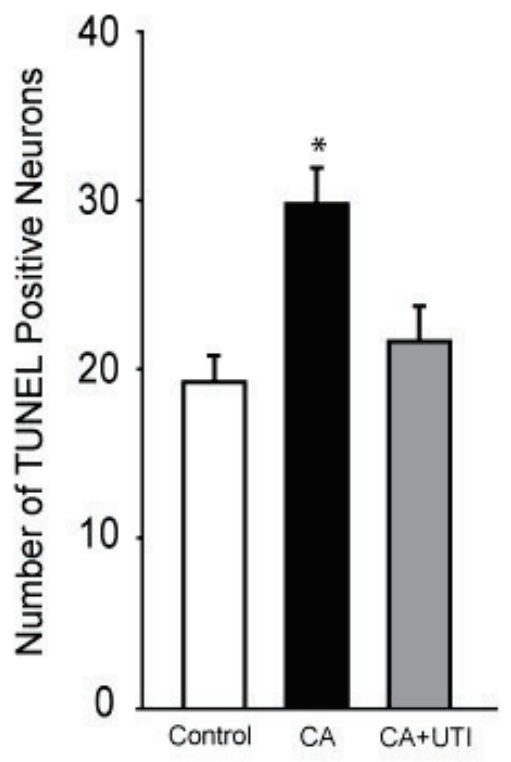

Fig. 2. Oxidative product and apoptotic signal. (A, B) Induction of CA amplified the levels of oxidative product 8-iso PGF2a and Caspase-3/Caspase-9 in the hippocampus CA1 region. Systemic administration of UTI attenuated increases of 8-iso PGF2a, and upregulation of cleaved form of Caspase-3/Caspase- 9 in CA rats. $* P<0.05$ vs. control rats and CA rats injected with UTI. For measurement of 8-iso PGF2a, $n=12$ in control; $n=18$ in CA group; and $n=25$ in CA group with UTI. For protein expression of Caspase-3/Caspase-9, $n=6-8$ in each group. (C) Representative images and averaged data illustrating that a greater number of TUNELpositive neuronal cells appeared in the hippocampus CA1 region of CA rats as compared with control rats. UTI significantly attenuated an increase of TUNEL-positive neuronal cells in CA rats. There were no significant differences observed in the number of TUNEL positive neurons between control rats and CA rats with UTI. $* P<0.05$ vs. control rats and CA rats injected with UTI. The number of animals was 8-10 in each group. 
A

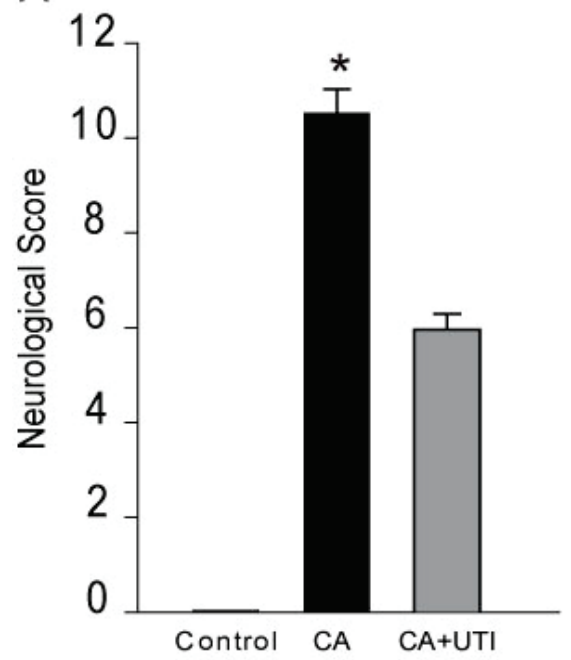

B

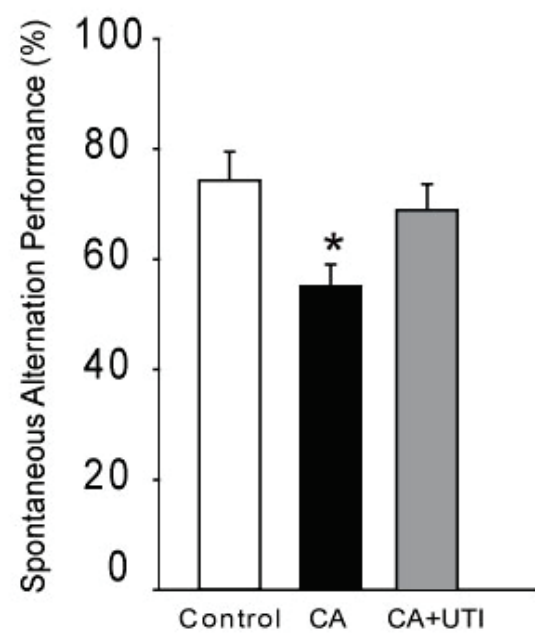

Fig. 3. Neurological functions. (A) The mNSS was increased in rats after CA and it was significantly improved by systemic administration of UTI. (B) Spatial working memory performance was decreased in CA rats and this was greatly restored by UTI. $* P<0.05$ vs. control rats and CA rats injected with UTI. The number of rats: $\mathrm{n}=12$ in control; $\mathrm{n}=18$ in CA group; and $n=25$ in CA group with UTI.
Figure $3 \mathrm{~A}$ shows that $\mathrm{CA}$ increased the $\mathrm{mNSS}$ in rats $(P<0.05$, control rats vs. CA rats; $\mathrm{n}=12$ in control and $\mathrm{n}=18$ in CA group). This figure further demonstrates that UTI significantly attenuated the impaired mNSS in animals following $\mathrm{CA} \quad(P<0.05, \mathrm{CA}$ rats with no treatment vs. CA rats with UTI; $n=25$ in CA group with UTI).

Spatial working memory performance was also assessed by recording spontaneous alternation performance. Likewise, Figure 3B shows that the percentage of spontaneous alternation was impaired in CA rats as compared with control rats. A decrease of spontaneous alternation was largely recovered in CA rats with injection of UTI. In addition, the number of arm entries was determined by counting the number of arms each animal entered in the maze during the test as a measure of activity level. No significant differences in the number of arm entries were observed among three groups. The number of arm entries for each group was: control rats $(n=12) 14.9 \pm 2.7$, CA rats $(n=18) 15.7 \pm 3.3$, and CA rats injected with UTI $(n=25) \quad 15.2 \pm 3.5(P>0.05$ among three groups).

\section{Discussion}

In the present study, we provided evidence showing that CA-induced transient cerebral ischemia upregulates autophagy protein expression, namely Atg5, LC3 and Beclin 1 in the hippocampus CA1. A decrease in the levels of p62 protein in the hippocampus of CA rats was also evidenced to support the engagement of autophagy. Importantly, amplification of the autophagy protein expression was attenuated in $\mathrm{CA}$ rats following systemic administration of UTI. Moreover, in the present study we found that CA-induced cerebral ischemia impairs the protein expression of Nrf2-ARE signal in the hippocampus of CA rats. It is noted that UTI can largely recover impairment of Nrf2-ARE following CA.

Under normal or unstressed conditions, Nrf2 is kept in the cytoplasm by a cluster of proteins that degrade it quickly (Itoh et al. 1997, Yamamoto et al. 2008, Sekhar et al. 2010). Under oxidative stress, Nrf2 is not degraded, but it travels to the nucleus where Nrf2 binds to a DNA promoter and initiates transcription of anti-oxidative genes and their proteins (Itoh et al. 1997, Yamamoto et al. 2008, Sekhar et al. 2010). In the nucleus, Nrf2 combines (forms a heterodimer) with a small Maf protein and binds to the ARE in the upstream promoter region of many anti-oxidative genes, and initiates their transcription (Yamamoto et al. 2008). In the present study, our data show that CA impairs the protein expression levels of Nrf2-ARE pathway in the hippocampus. It was also observed that Nrf2 and NQO1 are not significantly altered by blocking autophagy per se although a production of reactive oxygen species (8-iso PGF2 $\alpha$ ) and apoptotic signals (Caspase-3 and Caspase-9) are effectively attenuated in the hippocampus by UTI. This indicates that protective mechanisms by Nrf2-ARE are likely independent of autophagy following cerebral ischemia. Nevertheless, we found that UTI can restore impaired levels of Nrf2-ARE. Thus, activation of Nrf2ARE pathway is implicated for benefits to ischemic brain injuries that are associated with inflammation and oxidative stress (Gold et al. 2012).

In our present study, we have demonstrated that UTI attenuated autophagy and Caspase-3/Caspase-9 and 
improved the $\mathrm{mNSS}$ in $\mathrm{CA}$ rats. We have further demonstrated that 3-MA effectively attenuated autophagy. Thus, we speculated that it would improve the worsen mNSS caused by cerebral ischemia if autophagy inhibitor 3-MA was applied.

A recent study has demonstrated that $\mathrm{CA}$ increases PICs including IL-1 $\beta$, IL-6 and TNF- $\alpha$ and upregulates their receptors IL-1R, IL-6R and TNFR1 in the hippocampus (Xing and $\mathrm{Lu}$ 2016). Systemic administration of hypoxia inducible factor-1 $\alpha$ (HIF-1 $\alpha$ ) stimulator or blocking HIF-1 $\alpha$ breakdown attenuates the exaggerated PIC signals in the hippocampus induced by CA (Xing and Lu 2016), and as a result neuronal apoptosis and neurological deficits are attenuated (Liu et al. 2016). This prior study has also shown that stabilizing HIF-1 $\alpha$ significantly attenuates increases of Caspase-3 and TUNEL evoked by induction of CA, and thus improves neurological deficits and neuronal edema (Liu et al. 2016). In particular, recent studies further suggest that systemic administration of UTI plays a protective role in regulating neurological deficits observed in CA rats via central PIC mechanisms (Sui et al. 2014, Jiang et al. 2016).

In the current study, we found that systemic injection of UTI attenuates amplification of Caspase-3 and Caspase-9 indicating cell apoptosis in the hippocampus CA1 region. As a result, UTI also decreases an increase of TUNEL positive staining, labeling of apoptotic cell. Data of our current study provide direct evidence suggesting that UTI is likely involved in neuroprotective effects and attenuating neuronal damages evoked by cerebral ischemia via apoptotic Caspase-3/ Caspase-9 signal pathway. Importantly, UTI can improve neurological severity score, learning performance and survival in CA rats. Thus, our data suggest a beneficial role played by UTI in modulating cerebral ischemia induced by $\mathrm{CA}$ and further indicate that autophagy, Nrf2-ARE and apoptosis mechanisms are involved in the effects of UTI.

It should be noted that UTI is a protease inhibitor and administration of UTI is likely to attenuate activity of protease in the present study. Since protease can activate proteinase-activated receptors (PARs; i.e. PAR2) (Cottrell et al. 2003), it is well reasoned that PAR2 may be involved in the effects of UTI observed in the present study after its administration.

In conclusion, transient cerebral ischemia induced by $\mathrm{CA}$ increases the protein expression levels of autophagy and apoptotic Caspase-3 and Caspase-9, and also impairs Nrf2-ARE signal in the hippocampus CA1 region. Systemic administration of UTI can attenuate amplification of autophagy such as Atg5, LC3 and Beclin 1 and Caspase-3 and Caspase-9, and further recover deficiency of Nrf2-ARE in ischemic animals. Thus, this improves neurological severity score, memory performance and survival in CA rats. Our data indicate the beneficial role played by UTI in alleviating cerebral ischemia reperfusion injury via autophagy, Nrf2-ARE and apoptosis mechanisms.

\section{Conflict of Interest}

There is no conflict of interest.

\section{Acknowledgements}

This study was supported by grants from the First Hospital of Jilin University (JDYY52015016), Norman Bethune Program of Jilin University (No. 2015335), Science and Technology Development Program of Jilin Province (20160520160JH), and grants from Health Department of Jilin Province (2012Z005) as well as from the National Natural Science Foundation of China (No. 81471830).

\section{Abbreviations}

3-MA - 3-methyladenine, 8-iso PGF2 $\alpha$ - 8-isoprostaglandin F2 $\alpha$, ARE - antioxidant response element, Atg5 - autophagy protein 5, CA - cardiac arrest, CPR cardiopulmonary resuscitation, HIF- $1 \alpha$ - hypoxia inducible factor-1 $\alpha$, LC3 - microtubule-associated protein 1A/ 1B-light chain 3, mNSS - modified neurological severity score, NQO1 - NADPH quinone oxidoreductase-1, Nrf2 nuclear factor (erythroid-derived 2)-like 2, PARs proteinase-activated receptors, PIC - pro-inflammatory cytokine, SQSTM1 - sequestosome 1, TUNEL - terminal deoxynucleotide transferase-mediated dUTP nick-end labeling, UTI - urinary trypsin inhibitor.

\section{References}

BLUMBERG RM, CADY EB, WIGGLESWORTH JS, MCKENZIE JE, EDWARDS AD: Relation between delayed impairment of cerebral energy metabolism and infarction following transient focal hypoxia-ischaemia in the developing brain. Exp Brain Res 113: 130-137, 1997. 
COTTRELL GS, AMADESI S, SCHMIDLIN F, BUNNETT N: Protease-activated receptor 2: activation, signalling and function. Biochem Soc Trans 31: 1191-1197, 2003.

CUI D, SHANG H, ZHANG X, JIANG W, JIA X: Cardiac arrest triggers hippocampal neuronal death through autophagic and apoptotic pathways. Sci Rep 6: 27642, 2016.

EDINGER AL, THOMPSON CB: Death by design: apoptosis, necrosis and autophagy. Curr Opin Cell Biol 16: 663-669, 2004.

GAO CJ, NIU L, REN PC, WANG W, ZHU C, LI YQ, CHAI W, SUN XD: Hypoxic preconditioning attenuates global cerebral ischemic injury following asphyxial cardiac arrest through regulation of delta opioid receptor system. Neuroscience 202: 352-362, 2012.

GOLD R, KAPPOS L, ARNOLD DL, BAR-OR A, GIOVANNONI G, SELMAJ K, TORNATORE C, SWEETSER MT, YANG M, SHEIKH SI, DAWSON KT: Placebo-controlled phase 3 study of oral BG-12 for relapsing multiple sclerosis. N Engl J Med 367: 1098-1107, 2012.

INOUE K, TAKANO H: Urinary trypsin inhibitor as a therapeutic option for endotoxin-related inflammatory disorders. Expert Opin Investig Drugs 19: 513-520, 2010.

INOUE K, TAKANO H, YANAGISAWA R, YOSHIKAWA T: Protective effects of urinary trypsin inhibitor on systemic inflammatory response induced by lipopolysaccharide. J Clin Biochem Nutr 43: 139-142, 2008.

ITOH K, CHIBA T, TAKAHASHI S, ISHII T, IGARASHI K, KATOH Y, OYAKE T, HAYASHI N, SATOH K, HATAYAMA I, YAMAMOTO M, NABESHIMA Y: An Nrf2/small Maf heterodimer mediates the induction of phase II detoxifying enzyme genes through antioxidant response elements. Biochem Biophys Res Commun 236: 313-322, 1997.

JIANG XM, HU JH, WANG LL, MA C, WANG X, LIU XL: Effects of ulinastatin on global ischemia via brain pro-inflammation signal. Translat Neurosci 7: 158-163, 2016.

KARNAD DR, BHADADE R, VERMA PK, MOULICK ND, DAGA MK, CHAFEKAR ND, IYER S: Intravenous administration of ulinastatin (human urinary trypsin inhibitor) in severe sepsis: a multicenter randomized controlled study. Intensive Care Med 40: 830-838, 2014.

KOIKE M, SHIBATA M, TADAKOSHI M, GOTOH K, KOMATSU M, WAGURI S, KAWAHARA N, KUIDA K, NAGATA S, KOMINAMI E, TANAKA K, UCHIYAMA Y: Inhibition of autophagy prevents hippocampal pyramidal neuron death after hypoxic-ischemic injury. Am J Pathol 172: 454-469, 2008.

LI L, CHEN J, SUN S, ZHAO J, DONG X, WANG J: Effects of estradiol on autophagy and Nrf-2/ARE signals after cerebral ischemia. Cell Physiol Biochem 41: 2027-2036, 2017.

LI X, SU L, ZHANG X, ZHANG C, WANG L, LI Y, ZHANG Y, HE T, ZHU X, CUI L: Ulinastatin downregulates TLR4 and NF-kB expression and protects mouse brains against ischemia/reperfusion injury. Neurol Res 39: 367-373, 2017.

LIU X, LU J, XING J: Stabilization of HIF-1 $\alpha$ modulates VEGF and Caspase-3 in hippocampus of rats following transient global ischemia induced by asphyxial cardiac arrest. Life Sci 151: 243-249, 2016.

LU Q, HARRIS VA, KUMAR S, MANSOUR HM, BLACK SM: Autophagy in neonatal hypoxia ischemic brain is associated with oxidative stress. Redox Biol 6: 516-523, 2015.

MOI P, CHAN K, ASUNIS I, CAO A, KAN YW: Isolation of NF-E2-related factor 2 (Nrf2), a NF-E2-like basic leucine zipper transcriptional activator that binds to the tandem NF-E2/AP1 repeat of the beta-globin locus control region. Proc Natl Acad Sci U S A 91: 9926-9930, 1994.

NOLAN JP, NEUMAR RW, ADRIE C, AIBIKI M, BERG RA, BÖTTIGER BW, CALLAWAY C, CLARK RSB, GEOCADIN RG, JAUCH EC, KERN KB, LAURENT I, LONGSTRETH WT, MERCHANT RM, MORLEY P, MORRISON LJ, NADKARNI V, PEBERDY MA, RIVERS EP, RODRIGUEZ-NUNEZ A, SELLKE FW, SPAULDING C, SUNDE K, HOEK TV: Post-cardiac arrest syndrome: epidemiology, pathophysiology, treatment, and prognostication. A Scientific Statement from the International Liaison Committee on Resuscitation; the American Heart Association Emergency Cardiovascular Care Committee; the Council on Cardiovascular Surgery and Anesthesia; the Council on Cardiopulmonary, Perioperative, and Critical Care; the Council on Clinical Cardiology; the Council on Stroke. Resuscitation 79: 350-379, 2008.

NORTHINGTON FJ, CHAVEZ-VALDEZ R, MARTIN LJ: Neuronal cell death in neonatal hypoxia-ischemia. Ann Neurol 69: 743-758, 2011. 
SEKHAR KR, RACHAKONDA G, FREEMAN ML: Cysteine-based regulation of the CUL3 adaptor protein Keap1. Toxicol Appl Pharmacol 244: 21-26, 2010.

SUI B, LI Y, MA L: Postconditioning improvement effects of ulinastatin on brain injury following cardiopulmonary resuscitation. Exp Ther Med 8: 1301-1307, 2014.

WANG G, WEN J, WILBUR RR, WEN P, ZHOU SF, XIAO X: The effect of somatostatin, ulinastatin and Salvia miltiorrhiza on severe acute pancreatitis treatment. Am J Med Sci 346: 371-376, 2013.

XING J, LU J: HIF-1alpha activation attenuates IL-6 and TNF-alpha pathways in hippocampus of rats following transient global ischemia. Cell Physiol Biochem 39: 511-520, 2016.

YAMAMOTO T, SUZUKI T, KOBAYASHI A, WAKABAYASHI J, MAHER J, MOTOHASHI H, YAMAMOTO M: Physiological significance of reactive cysteine residues of Keap1 in determining Nrf2 activity. Mol Cell Biol 28: 2758-2770, 2008. 